\title{
INFORMACIÓN SOBRE
} LA SEGURIDAD DE LOS MEDICAMENTOS QUE CONSUMEN LOS PACIENTES DE LA OBRA SOCIAL UNIVERSITARIA, EN CÓRDOBA ARGENTINA.

\author{
INFORMATION ABOUT SAFETY OF \\ DRUGS TAKEN BY PATIENTS WHO HAVE \\ UNIVERSITY HEALTH INSURANCE, IN \\ CORDOBA, ARGENTINA .
}

Graciela A. Nievas, ${ }^{1,2}$

Ana C. López,2,3 Mariana Caffaratti ${ }^{4}$

${ }^{1}$ Estudiante de la Maestría en Salud Pública de la Universidad Autónoma de Nayarit, estancia profesional en la

Universidad Nacional de Córdoba : alejandra.nievas@ uan.edu.mex

2DASPU Obra Social Universitaria de Córdoba, Argentina

${ }^{3}$ Facultad de Ciencias Médicas, Universidad Nacional de Córdoba ${ }^{4}$ Centro de Información de Medicamentos, Facultad de Ciencias Quimicas Universidad Nacional de Córdoba

Autor de contacto: Dra.Ana C López analopez.af@gmail.

Trabajo recibido: 11 de abril 2019.

Aprobado: 10 de junio
INFORMAÇÕES SOBRE A SEGURANÇA DOS MEDICAMENTOS CONSUMIDOS PELOS PACIENTES DA ASSISTÊNCIA SOCIAL UNIVERSITÁRIA, EM CÓRDOBA, ARGENTINA.

\section{Resumen}

Objetivos: Describir la información sobre la seguridad de los medicametos que cosumen los pacientes afiliados a la Obra Social Universitaria y conocer sí los pacientes tenían interés en recibir información sobre estos medicamentos. Métodos: Estudio descriptivo, observacional y de corte transversal, con una muestra no probabilística (por conveniencia) de $\mathrm{n}=81$ de los pacientes que acudieron a dispensar su medicamento a la farmacia central de la Obra Social Universitaria, se utilizó un cuestionario validado.Resultados: El 64\% de los encuestados fueron del sexo masculino, con una media de edad de 57,22 \pm 1,36. El $63 \%$ alcanzó un nivel de instrucción universitario, siendo el $36 \%$ jubilado, además el $32 \%$ de los encuestados solicito información sobre el medicamento que consumía. En los aspectos evaluados por el cuestionario como el conocimiento sobre las precauciones respecto al medicamento, el $83 \%$ respondió de manera incorrecta o dijo no conocer ninguna precaución.

Palabras clave: Conocimiento del medicamento, $\mathrm{Pa}$ ciente, Conocimiento. 
Objectives: To describe information about the safety of medicine taken by patients who have the university health insurance and know if these patients were interested in receiving this information. Methods: Descriptive, observational, cross-sectional study with nonprobabilistic sample (for convenience) of $n=81$ of patients who went to the University Health Insurance central chemist to be dispensed their medicine; a validated questionnaire was used. Results: $64 \%$ of the respondents were male with average age of $57.22 \pm 1.36$, $63 \%$ reached university level of education, $36 \%$ were retired, besides, $32 \%$ requested information on the drugs they were taking. In aspects evaluated in the questionnaire such as knowledge about precautions regarding the drug, 83\% answered incorrectly or said they did not know about any precaution.

Key words: Knowledge about the drugs; Patient; Knowledge.

Resumo

Objetivos: Descrever as informações sobre a segurança dos medicamentos consumidos pelos pacientes afiliados ao Serviço Social da Universidade e saber se eles estavam interessados em receber informações sobre esses medicamentos Métodos: Estudo descritivo, observacional e transversal, através de uma amostra não probabilística (por conveniência) de $\mathrm{n}=81$ dos pacientes que adquiriram seus medicamentos na farmácia central do Serviço Social da Universidade, foi utilizado um questionário validado. Resultados: 64\% dos entrevistados eram do sexo masculino, com média 57,22 $\pm 1,36$. 63\% atingiram um nível de ensino superior, $36 \%$ aposentados e $32 \%$ dos entrevistados solicitaram informações sobre os medicamentos que usavam. Nos aspetos avaliados pelo questionário, como conhecimento sobre as precauções em relação à medicação, $83 \%$ responderam incorretamente ou afirmaram não conhecer qualquer precaução.

Palavras chave: Conhecimento do medicamento, Paciente, Conhecimento.

\section{Introducción}

La acción de prescribir medicamentos es el acto médico más frecuente. Por consecuente, el uso de estos está presente en la vida diaria de muchas personas que tienen acceso a ellos.Sin embargo, la falta de información o la información incorrecta, así como la mala interpretación u olvido de las instrucciones que el paciente ha recibido, puede ocasionar diversos problemas como la disminución de la efectividad de estos y el uso incorrecto de los mismos(1-3).

De hecho, la morbimortalidad relacionada al uso de medicamentos se ha convertido en un problema de salud pública, siendo las Reacciones Adversas al Medicamento (RAM) las consecuencias más serias al uso inadecuado de estos, ya que tienen un alto costo clínico, social y económico. En algunos países las RAM son entre la cuarta y la sexta causa de muerte(4,5).

Por su parte, desde 1968 la Organización Mundial de la Salud (OMS) propuso la creación de un centro para la farmacovigilancia internacional establecido en Uppsala, Suecia (Centro de Monitoreo de Uppsala, o UMC, por sus siglas en inglés). Muchos países cuentan con sistemas de farmacovigilancia los cuales están incorporados al sistema internacional de la OMS. Argentina fue admitida como país miembro en 1994, y en el 2005 Córdoba, una provincia de este país creó el Sistema Unificado de Farmacovigilancia(6,7).

En el 2016 el Sistema Nacional de Farmacovigilancia (SNFVG) de Argentina, recibió y analizó 10,597 notificaciones iniciales ingresadas a través del formulario en linea para reportes válidos de las RAM. Por la misma vía se recibieron 2,356 reportes de seguimiento 
y por expedientes semestrales casos desestimados y no relacionados 3452 casos. En Córdoba se registraron 257 casos(8).

En la actualidad, poco se ha explorado científicamente la medida en que el conocimiento o la información correcta que posee el paciente sobre el medicamento que consume puede prevenir o disminuir la severidad de una RAM.Se podría esperar que los pacientes que estén bien informados pudieran evitar interacciones, reconocer adecuadamente las posibles RAM y tener un mejor manejo en todo lo referente al consumo de sus medicamentos(7). Los objetivos de este trabajo fueron describir la información sobre la seguridad de los medicamentos que cosumen los pacientes afiliados a la Obra Social Universitaria y conocer si los pacientes tenían interés en recibir información sobre estos medicamentos.

\section{Material y Métodos}

Se llevó a cabo un estudio descriptivo, observacional y de corte transversal, con una muestra no probabilística (por conveniencia) de $n=81$, de los pacientes que acudieron en el mes de septiembre a buscar su medicamento a la farmacia central de la Obra Social Universitaria de la provincia de Córdoba. Se incluyeron pacientes de ambos sexos, mayores de 18 años, que acudieron a la farmacia por su medicamento y que aceptaron participar en el proyecto de investigación y fueron excluídos los pacientes con problemas cognitivos, menores de edad, y las personas que fueron a la farmacia a comprar otro tipo de artículos que no eran medicamentos (artículos de higiene personal y otros), así como aquellas que se negaron a responder y/o a participar en el estudio.

El instrumento que se utilizó fue un cuestionario validado por García-Delgado y colaboradores que mide la información que tienen los pacientes sobre el medicamento que consumen mediante 11 ítems que evalúan los aspectos de indicaciones, pauta/posología, forma de administración, duración del tratamiento, efectividad del tratamiento, precauciones, condiciones de conservación, contraindicaciones, interacciones y efectos secundarios.

Los pacientes que aceptaron participar respondiendo el cuestionario lo hacían respecto al medicamento que tenían prescrito en su receta médica, si los pacientes tenían más de un medicamento, se seleccionaba un medicamento al azar.

Además, los pacientes tuvieron la opción de elegir si deseaban recibir información sobre los medicamentos que consumían. Los que respondieron afirmativamente que sí la deseaban, llenaron una solicitud de información del Centro de Información de Medicamentos de Ciencias Químicas de la Universidad Nacional de Córdoba, donde se procesó la solicitud y se dio respuesta.

Los resultados se procesaron en el programa SPSS versión 25. La valoración de las contestaciones como correctas o incorrectas, se contrastaron con información científica de referencia. Los informes solicitados por los pacientes, se elaboraron mediante la utilización de diversas fuentes,siendo la principal Medline Plus, AHFS ${ }^{\circledR}$ Consumer Medication Information de la Biblioteca Nacional de Medicina de Estados Unidos, además de usar los prospectos de la Agencia Española del Medicamento y Productos Sanitarios cuando los medicamentos no fueron encontrados en Medline Plus.

\section{Resultados}

Cerca de dos tercios de los participantes (63\%) declararon tener un nivel de estudios universitario completo, $36 \%$ eran jubilados, y la media de edad fue de 57.22 años. Respecto al número de medicamentos que tomaban más del 30\% de los encuestados señalo estar consumiendo de 3 a 4 medicamentos (Tabla 1). 
Tabla $\mathbf{N}^{0}$ 1: Características sociodemográficas de la población y características del medicamento que consume.

\begin{tabular}{|c|c|c|}
\hline Variable & Frecuencia & Porcentaje \\
\hline \multicolumn{3}{|l|}{ Género } \\
\hline Masculino & 52 & $64 \%$ \\
\hline Femenino & 29 & $36 \%$ \\
\hline \multicolumn{3}{|l|}{ Nivel de estudios } \\
\hline Primario incompleto & 1 & $1 \%$ \\
\hline Primario completo & 1 & $1 \%$ \\
\hline Secundario incompleto & 2 & $3 \%$ \\
\hline Secundario completo & 10 & $12 \%$ \\
\hline Universitario incompleto & 8 & $10 \%$ \\
\hline Universitario completo & 51 & $63 \%$ \\
\hline Estudiante Universitario & 8 & $10 \%$ \\
\hline \multicolumn{3}{|l|}{ Ocupación } \\
\hline Jubilado & 29 & $36 \%$ \\
\hline Ama de casa & 17 & $21 \%$ \\
\hline Empleado & 10 & $12 \%$ \\
\hline Docente & 6 & $7 \%$ \\
\hline Comerciante & 9 & $11 \%$ \\
\hline Varios (contador, arquitecto) & 8 & $10 \%$ \\
\hline Profesionales de la Salud & 2 & $3 \%$ \\
\hline \multicolumn{3}{|l|}{$\begin{array}{l}\text { Años que lleva tomando el } \\
\text { medicamento }\end{array}$} \\
\hline $0-5$ & 53 & $65 \%$ \\
\hline $6-10$ & 21 & $26 \%$ \\
\hline $11-15$ & 3 & $4 \%$ \\
\hline $16-20$ & 1 & $1 \%$ \\
\hline 21 años o más & 3 & $4 \%$ \\
\hline \multicolumn{3}{|l|}{ Número de medicamentos } \\
\hline $1-2$ & 22 & $27 \%$ \\
\hline 3-4 & 31 & $38 \%$ \\
\hline 5 o más & 28 & $35 \%$ \\
\hline \multicolumn{3}{|l|}{$\begin{array}{l}\text { Preocupación por el } \\
\text { problema de salud }\end{array}$} \\
\hline Bastante & 34 & $42 \%$ \\
\hline Regular & 30 & $37 \%$ \\
\hline Poco & 17 & $21 \%$ \\
\hline
\end{tabular}

Respecto a los ítems de la seguridad del medicamento, el conocimiento de las precauciones que se deben tener sobre el consumo del medicamento el $83 \%$ respondió de manera incorrecta, refirió no saber o no conocer ninguna; sobre contraindicaciones el $90 \%$ respondió de manera incorrecta, refirió no saber o no conocer ninguna contraindicación o que no existía con ese medicamento; en lo referente al conocimiento sobre las interacciones del medicamento el $89 \%$ respondió de manera incorrecta refiriendo no saber, no conocer ninguna, o que no existían interacciones medicamentosas con ese medicamento; respecto a las RAM el 83\% contesto de manera incorrecta, refiriendo no saber, no conocer ninguna RAM, o que no existían RAM con ese medicamento y el $17 \%$ contesto de manera correcta; los que contestaron de manera correcta se les pregunto acerca de cómo había obtenido la información y el 74\% lo sabía porque las había experimentado. (Tabla 2). 
Tabla $\mathbf{N}^{\mathbf{0}}$ 2: Distribución del grado de conocimiento del paciente para los items de seguridad del medicamento que consume.

\begin{tabular}{|c|c|c|}
\hline Variable & Frecuencia & Porcentaje \\
\hline \multicolumn{3}{|l|}{ Precauciones } \\
\hline Incorrecta/NS/NE/NC & 67 & $83 \%$ \\
\hline Correcta & 14 & $17 \%$ \\
\hline \multicolumn{3}{|l|}{ Contraindicaciones } \\
\hline Incorrecta/NS/NE/NC & 73 & $90 \%$ \\
\hline Correcta & 8 & $10 \%$ \\
\hline \multicolumn{3}{|l|}{ Interacciones } \\
\hline Incorrecta/NS/NE/NC & 72 & $89 \%$ \\
\hline Correcta & 9 & $11 \%$ \\
\hline \multicolumn{3}{|l|}{ RAM } \\
\hline Incorrecta/NS/NE/NC & 67 & $83 \%$ \\
\hline Correcta & 14 & $17 \%$ \\
\hline \multicolumn{3}{|l|}{ Conocimiento de las RAM } \\
\hline Experiencia propia con RAM & 10 & $74 \%$ \\
\hline $\begin{array}{l}\text { Alguien más le proporciono la } \\
\text { información, la leyó en el } \\
\text { prospecto o lo invetigó en } \\
\text { internet }\end{array}$ & 4 & $26 \%$ \\
\hline
\end{tabular}

Referente a las patologías para lo que utilizaba el paciente al medicamento el porcentaje mayor fue: enfermedades del sistema circulatorio con un 35\% (Tabla 3).

Tabla $N^{0}$ 3: Enfermedad para lo que utilizaban su medicamento

\begin{tabular}{|c|c|c|}
\hline Patología & Frecuencia & Porcentaje \\
\hline $\begin{array}{l}\text { Enfermedades del sistema } \\
\text { circulatorio }\end{array}$ & 28 & $35 \%$ \\
\hline Enfermedades endocrinas & 17 & $21 \%$ \\
\hline $\begin{array}{l}\text { Enfermedades del sistema } \\
\text { respiratorio }\end{array}$ & 10 & $12 \%$ \\
\hline $\begin{array}{c}\text { Enfermedades del sistema } \\
\text { digestivo }\end{array}$ & 8 & $10 \%$ \\
\hline $\begin{array}{c}\text { Enfermedades del sistema } \\
\text { nervioso }\end{array}$ & 4 & $5 \%$ \\
\hline Traumatismos & 4 & $5 \%$ \\
\hline $\begin{array}{c}\text { Enfermedades del sistema } \\
\text { genitourinario }\end{array}$ & 4 & $5 \%$ \\
\hline Trastornos mentales & 2 & $3 \%$ \\
\hline
\end{tabular}

De los pacientes que accedieron a contestar el cuestionario, el 32\% solicitaron recibir información acerca de los medicamentos que consumían, siendo el mayor porcentaje el sexo masculino con un $65 \%$. La preferencia de los pacientes para recibir el/los informes solicitados fue el correo electrónico (medio más solicitado) 85\% ( Tabla 4). 
Tabla $\mathbf{N}^{0}$ 4: Número de informes solicitados por el paciente

\begin{tabular}{|c|c|c|}
\hline Variable & Frecuencia & Porcentaje \\
\hline \multicolumn{3}{|l|}{ Sexo } \\
\hline Masculino & 17 & $65 \%$ \\
\hline Femenino & 9 & $35 \%$ \\
\hline \multicolumn{3}{|l|}{$\begin{array}{l}\text { Preferencia respecto a } \\
\text { recibir los informes }\end{array}$} \\
\hline Correo electrónico & 22 & $85 \%$ \\
\hline Impreso & 4 & $15 \%$ \\
\hline \multicolumn{3}{|l|}{$\begin{array}{l}\text { Número de informes } \\
\text { solicitados }\end{array}$} \\
\hline 1 Informe & 13 & $50 \%$ \\
\hline 2 Informes & 6 & $23 \%$ \\
\hline 3 Informes o más & 7 & $27 \%$ \\
\hline
\end{tabular}

\section{Discusión}

La falta de información o la información incorrecta que tienen los pacientes sobre sus medicamentos, se pudo comprobar con este trabajo. Los resultados son similares al trabajo publicado por Rubio y colaboradores donde el porcentaje de pacientes que contestaron fueron en su mayoría jubilados/ama de casa. Así mismo, los resultados de las contraindicaciones son muy similares a los reportados por Clavel Rojo, ya que el porcentaje fue de un $96 \%$ de los pacientes que dijeron no saber o no conocer la respuesta o que contestaron de manera incorrecta. Respecto a las RAM los resultados obtenidos en este estudio son comprables a los obtenidos por Caffaratti donde las RAM fueron las que obtuvieron el porcentaje de respuesta más alto sobre las preguntas de seguridad con un $17.8 \%$ contestadas. La información sobre las precauciones los resultados de este estudio también son parecidos a los publicados por García-Delgado y colaboradores donde el $9.2 \%$ de los encuestados contestaron correctamente $(9,7,10,11)$.

El alto porcentaje de pacientes que declaraban no saber, o no conocían sobre los aspectos de seguridad de los medicamentos es elevado, pero también se tiene que recalcar las respuestas incorrectas que daban los pacientes. En estas preguntas, donde daban respuesta como "con este medicamento no existen ninguna contraindicación, "mi medicamento no lo puedo suspender por nada me lo tengo que tomar o sí o sí" dejan en evidencia esta información que es incorrecta, pero que el paciente cree que es correcta, lo que representa también un riesgo para la salud.

Sobre los pacientes que pidieron información de su medicamento, la mayoría prefirió recibir este documento vía email, esto difiere al estudio publicado por Brounéus et al, donde los pacientes preferían recibir la información de su medicamento personalmente por su médico o su farmacéutico, y también difiere del porcentaje de pacientes que solicitaron el informe fue menor al reportado por Caffaratti y Hamrosi $(12,13)$.

\section{Conclusiones}

La falta de información y/o la información incorrecta respecto a la seguridad de los medicamentos por parte de los pacientes que acudieron a la farmacia central de la Obra Social Universitaria es preocupante, puesto que indica que los pacientes tienen riesgos potenciales de salud que podrían ser prevenibles si tuvieran una información correcta sobre los medicamentos que consumen. Además, la población que accedió a participar en el proyecto y solicito información sobre los medicamentos que consumía se vio muy interesada en saber más referente a este tipo de intervenciones, lo cual indica que si sigue colaborando en conjunto con un Centro de Información de Medicamentos los resultados podrían ser más eficientes y por consecuente más alentadores para promover la información necesaria y adecuada para los pacientes respecto a uso de sus medicamentos. 
1. Zavala-González M, Cabrera-Pivaral C, Orozco-Valerio M, Ramos-Herrera I. Efectividad de las intervenciones para mejorar la prescripción de medicamentos en atención primaria. Aten Primaria [Internet]. 2017;49(1):13-20. Disponible en: fhttp://dx.doi.org/10.1016/j.aprim.2016.02.00

2. García-Delgado P, Gastelurrutia-Garralda M, Baena-Parejo M, Fisac- Lozano F, Martínez-Martínez F. Validación de un cuestionario para medir el conocimiento de los pacientes sobre sus medicamentos. Atención Primaria. 2009;41(12):6618. Disponible en : https://www.sciencedirect.com/science/article/pii/ S0212656709002637

3. Vidal M. Incumplimiento del tratamiento farmacologico. Inf Medicam al paciente y Mejor en el cumplimiento del Trat [Internet]. 2000;23:257-72. Disponible en: http://www.scielo.org.co/scielo.php?script=sci_arttext\&pid=S0034$74182006000200001 \&$ lang=pt

4. García Delgado P, Gastelurrutia Garralda MÁ, Baena Parejo MI, Fisac Lozano F, Martínez Martínez F. Validación de un cuestionario para medir el conocimiento de los pacientes sobre sus medicamentos. Aten Primaria. 2009;41(12):661-8.

5. Oscanoa T. Uso Inadecuado de Medicamentos en Adultos Mayores. Universidad Nacional Mayor de San Marcos. ISSN 1025 - 5583. An Fac Med Lima. [Internet].2005;66(1):43-52. Disponible en : https://www.redalyc.org/ pdf/379/37966107.pdf

6. Organización Panamericana de la Salud. Red PARF, Documento Técnico No.5: Buenas Prácticas de Farmacovigilancia para las Américas [Internet]. Washington. 2010. 78 p. Disponible en: http://apps.who.int/medicinedocs/documents/s18625es/ s18625es.pdf

7. Caffaratti M. Conocimiento de la seguridad de los medicamentos por parte de los pacientes. (Tesis de Maestría). Universidad Nacional de Cordobá.[Internet]. 2017. Disponible en:http://lildbi.fcm.unc.edu.ar/lildbi/tesis/Caffaratti-mariana-2017.pdf

8. Departamento de Farmacovigilancia Admnistración de Medicamentos Alimentos y Tecnología Médica anmat . Informe Anual 2016 2016;1-56. Disponible en: https:// www.argentina.gob.ar/sites/default/files/farmacovigilancia-informe_2016.pdf

9. Clavel Rojo A. Conocimiento del paciente acerca de la medicación prescrita: influencia de las fuentes de información y legibilidad de los prospectos. (Tesis doctoral). Universidad de Murcia España.. Disponible en: https://digitum.um.es/ xmlui/handle/10201/35466.

10. Rubio JS, García-Delgado P, Iglésias-Ferreira P, Mateus-Santos H, MartínezMartínez F. Measurement of patients' knowledge of their medication in community pharmacies in Portugal. Cien Saude Colet [Internet]. 2015;20(1):219-28. Disponible en : http://www.scielo.br/scielo.php?script=sci_arttext\&pid=S141381232015000100219\&lng=en\&tlng=en

11. García P Conocimiento del Paciente sobre sus Medicamentos.(Tesis doctorla). Universidad De Granada Facultad De Medicina [Internet]. 2012. 251 p.Disponible en : https://hera.ugr.es/tesisugr/18971647.pdf

12. Brouneus F, Macleod G, Maclennan K, Parkin L, Paul C. Drug safety awareness in New Zealand: public knowledge and preferred sources for information. J Prim Health Care. 2012;4(4):288-93. Disponible en: https://www.ncbi.nlm.nih.gov/ pubmed/23205377.

13. Hamrosi KK, Raynor DK, Aslani P. Pharmacist, general practitioner and consumer use of written medicine information in Australia: Are they on the same page? Res Soc Adm Pharm. 2014;10(4):656-68. Disponible en: http://eprints.whiterose. ac.uk/93954/7/RSAP-D-13-00150R1.pdf 